\title{
Instantaneous wavefront measurement based on deflectometry
}

Xie, Zhongming, Wang, Daodang, Gu, Hanting, Kong, Ming, Liang, Rongguang, et al.

Zhongming Xie, Daodang Wang, Hanting Gu, Ming Kong, Rongguang Liang, Wentao Zhang, "Instantaneous wavefront measurement based on deflectometry," Proc. SPIE 11185, Optical Design and Testing IX, 111850Q (19 November 2019); doi: 10.1117/12.2537148

SPIE. Event: SPIE/COS Photonics Asia, 2019, Hangzhou, China 


\title{
Instantaneous wavefront measurement based on deflectometry
}

\author{
Zhongmin Xie ${ }^{\mathrm{a}}$, Daodang Wang ${ }^{*}$, Hanting Gu ${ }^{\mathrm{a}}$, Ming Kong ${ }^{\mathrm{a}}$, Rongguang Liang ${ }^{\mathrm{b}}$, Wentao Zhang ${ }^{\mathrm{c}}$ \\ ${ }^{\mathrm{a}}$ College of Metrology and Measurement Engineering, China Jiliang University, Hangzhou 310018, \\ China; ${ }^{b}$ James C. Wyant College of Optical Sciences, University of Arizona, Tucson, Arizona 85721, \\ USA; 'Guangxi Key Laboratory of Optoelectronic Information Processing (Guilin University of \\ Electronic Technology), Guilin 541004, China
}

\begin{abstract}
The deflectometry enables high-precision wavefront measurement with large dynamic range. Traditional multi-step phase-shifting fringe-illumination deflectometric methods involve at least three sinusoidal phase-shifting fringe patterns and require a sequential projection, making it not feasible for the instantaneous measurement. In this paper, a colorcoded method with frequency-carrier patterns is proposed to achieve the instantaneous wavefront measurement based on deflectometry. With the color extraction from different channels, composite patterns in $x$ and $y$ directions can be well separated with a single shot. Then, the phase-shifting patterns encoded in different frequencies can be demodulated with the designed filters, by which the local wavefront slopes can be obtained simultaneously to reconstruct the wavefront under test. Both the numerical simulation and experiments are performed to validate the feasibility of proposed method. The proposed method provides a feasible way for the real-time and instantaneous measurement with large dynamic range based on deflectometry.
\end{abstract}

Keywords: instantaneous deflectometry; color-coded method; fringe illumination; frequency carrier

\section{INTRODUCTION}

The testing of transmitted wavefronts provides an effective and quantitative approach to evaluate the transmission elements, such as the refractive optics, transparent glass bottles and even flow field. Traditionally, the wavefront testing is achieved with interferometry [1-4], including Twyman-Green interferometer, Fizeau interferometer and pointdiffraction interferometer, etc. Generally, the interferometers are complex in configuration, high in cost and of inflexibility. Though ultra-high testing precision can be obtained with interferometry, its dynamic range is quite limited, making it not suitable for testing the highly distorted wavefronts [5]. As a slope-based measurement technique, the deflectometry provides a feasible way to achieve accurate wavefront testing with large dynamic range, with extremely simple system configuration.

In the past few years, various deflectometric methods and systems have been proposed to achieve accurate optical testing, including the astronomical telescope mirrors [6], spherical surfaces [7], aspheric mirrors [8, 9], specular freeform mirrors [10] and precision $x$-ray mirrors [11]. Compared to interferometrical methods, the deflectometry is much lower in cost due to its simple system configuration, simply consisting of a projection screen, CCD camera and test object. In the deflectometric testing, the ray tracing is generally required to determine the rays from the camera through the object to the screen, by which the test wavefront can be obtained from the ray aberration. The projection patterns, such as binary patterns, gray-level patterns, phase shifting patterns and hybrid method, can be used to create the mapping to achieve the deflectometric testing [12]. The multi-step phase-shifting method, in which the projection screen displays the phaseshifted sinusoidal patterns in orthogonal directions, is commonly used for the general testing. However, this method involves at least three sinusoidal phase-shifting fringe patterns in each direction and requires a sequential projection, making it not feasible for the instantaneous measurement. To meet the requirement of instantaneous testing with a single shot, the Fourier transform profilometry (FTP) [13] and instantaneous coding method with color channels [14] have been developed. The real-time testing with an instantaneous phase-shifting deflectometry [15], which is based on color-coded multiplexing phase-shifting fringe patterns, has been successfully achieved. However, the wide spectral range in display could result in a residual error in the phase-shifting calculation; besides, the crosstalk among RGB channels and photon

*wangdaodang@sina.com; phone 86-571-86914563

Optical Design and Testing IX, edited by Yongtian Wang, Pablo Benítez,

Osamu Matoba, Proc. of SPIE Vol. 11185, 111850Q · C 2019 SPIE

CCC code: $0277-786 \mathrm{X} / 19 / \$ 21 \cdot$ doi: $10.1117 / 12.2537148$ 
response nonuniformity would also introduce an obvious additional measurement error, and it requires complex calibration.

In this paper, an instantaneous phase-shifting deflectometry based on frequency carrier composite is presented to achieve accurate wavefront testing. In this method, the multi-step phase-shifting patterns in orthogonal directions are encoded into a single composite color pattern with different frequency carriers. Based on the designed demodulation algorithm, the multiple patterns can be retrieved from the captured composite pattern after passing through the testing object with a single shot. With the retrieved patterns, the phase-shifting deflectometry can be applied to reconstruct the test wavefront, based on which the transmission elements can be quantitatively evaluated. Section 2 presents the principle of the proposed instantaneous deflectometry. In Sections 3 and 4, numerical simulation and experimental results are given to demonstrate the feasibility of the proposed method. Some conclusions are drawn in Section 5.

\section{PRINCIPLE}

\subsection{Principle of deflectometric wavefront measurement}

In the traditional fringe-illumination deflectometry, at least three sinusoidal phase-shifting fringes in each direction are required to be sequentially projected on the test object, and it enables accurate wavefront measurement with large dynamic range. Figure 1 shows the schematic diagram of traditional deflectometry with minimum system configuration for transmission surface testing. The screen is used to project the specific designed patterns and the camera to capture the distorted patterns passing through testing object. The designed patterns and the corresponding images captured by camera can be applied to create a mapping between the coordinates on the screen and camera. Traditionally, the phaseshifted sinusoidal patterns with fixed phase shift covering a full $2 \pi$ period in $x$ and $y$ directions are projected on test object to create the mapping of this system. Based on the mapping, the actual spot distribution $\left(x_{\text {test }}, y_{\text {test }}\right)$ in the experiment can be obtained, and the corresponding ideal spot distribution $\left(x_{\text {model }}, y_{\text {model }}\right)$ on the imaging plane is obtained by ray tracing the modeled testing system [16]. Based on transverse ray aberration model, the system wavefront aberration $W(x, y)$ can be calculated according to the slope differences $\left(\Delta w_{x}, \Delta w_{y}\right)$ [17], and we have

$$
\left\{\begin{array}{l}
\Delta w_{x}=\frac{\partial W(x, y)}{\partial x}=-\frac{\Delta x}{d_{\mathrm{L} 2 S}}, \\
\Delta w_{y}=\frac{\partial W(x, y)}{\partial y}=-\frac{\Delta y}{d_{\mathrm{L} 2 S}}
\end{array}\right.
$$

where $(x, y)$ is the coordinate of system exit pupil, $(\Delta x, \Delta y)$ can be defined as $\Delta x=x_{\text {test }}-x_{\text {model }}$ and $\Delta y=y_{\text {test }}-y_{\text {model }} ; d_{\mathrm{L} 2 S}$ is the measured distance between test surface and illumination screen. With the slope differences $\left(\Delta w_{x}, \Delta w_{y}\right)$, the transmitted wavefront aberration from test object can be reconstructed according to the integration method.

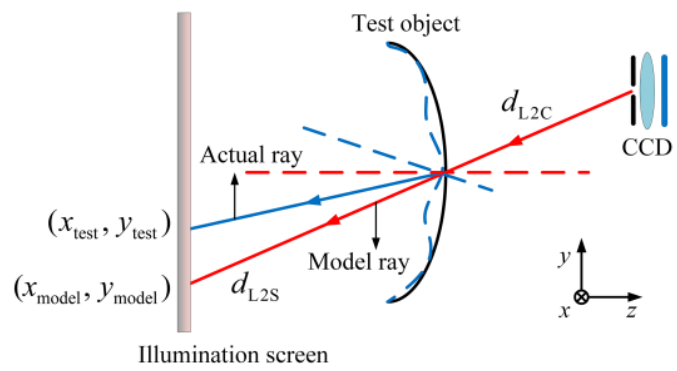

Fig. 1. Schematic diagram of traditional deflectometric wavefront testing.

\subsection{Frequency-carrier composite color pattern for instantaneous deflectometric measurement}

Figure 2 shows the generation of the composite pattern combining the color channels, in which the four-step (instead of three-step) phase-shifting fringes are used to reduce the sensitivity of the multi-step algorithm to the phase shift error [18]. In order to combine both the multiple phase-shifting patterns in $x$ and $y$ directions to achieve single-shot testing, the frequency carrier stripes in each direction are utilized to modulate the individual phase-shifting pattern, and the phaseshifting combined composite patterns in orthogonal directions are coupled into different color channels. 


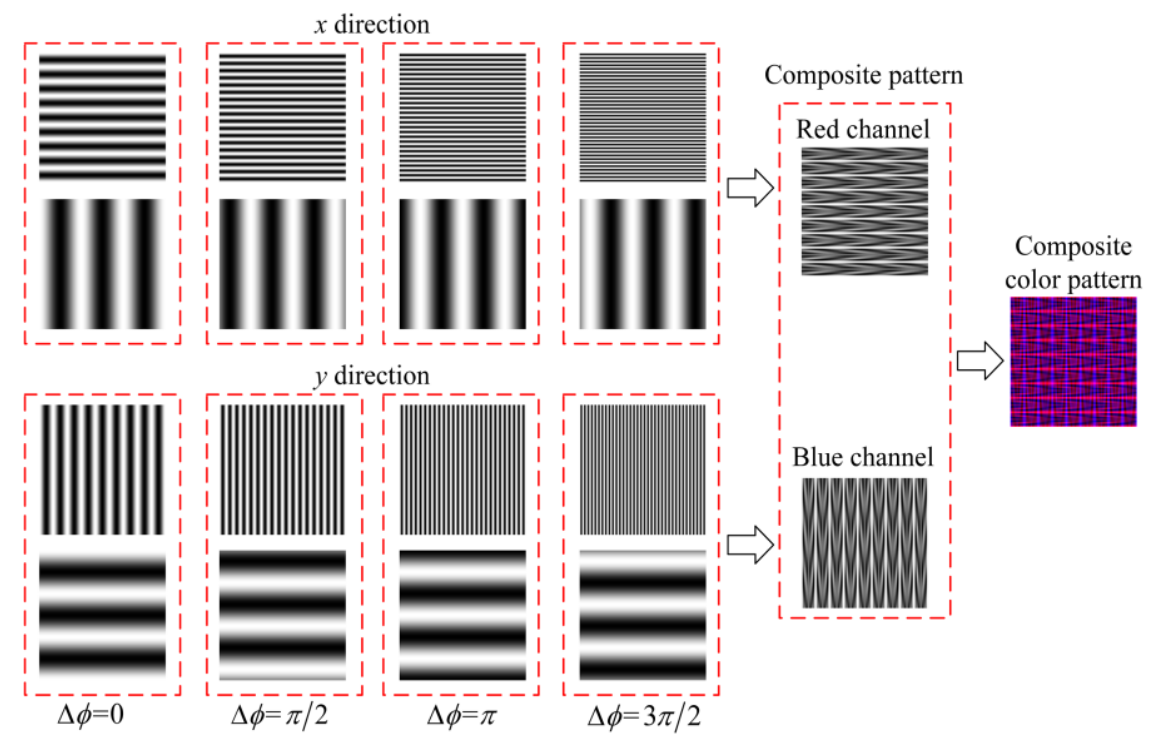

Fig. 2. Overview of the designed frequency-carrier composite color pattern for instantaneous deflectometric measurement.

The phase-shifting pattern in $x$ direction can be expressed by

$$
I_{p x, n}=1 / 2+\cos (2 \pi f x+n \cdot \pi / 2),
$$

where $f$ is the frequency of phase-shifting patterns, $x$ is the pixel coordinates on illumination screen, and $n$ is the phaseshifting step $(n=1,2,3,4)$. The distinct frequency carrier patterns in each direction are applied to modulate the phaseshifting patterns, and the modulated patterns are then superposed into a single pattern,

$$
I_{C x}=A+B \cdot \sum_{n=1}^{4}\left(I_{p x, n} \cdot I_{f x, n}\right),
$$

where $I_{f x, n}=\cos \left(2 \pi f_{f x, n} y\right)$ refers the distinct frequency carrier patterns, $f_{f x, n}$ is the designed frequency of each carrier pattern, $A$ and $B$ are designed to fulfill the intensity of projection screen by the composite pattern. It should be noted out that the $f_{f x, n}$ are designed away from zero frequency. Similarly, the phase-shifting composite pattern of in $y$ direction can be designed in the same way according to Eqs. (2) and (3). Then, the two composite patterns in orthogonal directions are coupled into two ( $\mathrm{R}$ and $\mathrm{B}$ ) color channels, respectively. The composite color pattern for projection can be mathematically expressed as

$$
\left\{\begin{array}{l}
I_{R}=I_{C x}=A+B \cdot \sum_{n=1}^{4}\left(I_{p x, n} \cdot I_{f x, n}\right) \\
I_{B}=I_{C y}=A+B \cdot \sum_{n=1}^{4}\left(I_{p y, n} \cdot I_{f y, n}\right)
\end{array},\right.
$$

where $I_{R}$ and $I_{B}$ are intensity in the R and B channels. With the composite color pattern in Eq. (4), the phase-shifting deflectometric measurement can be achieved with a single shot.

\subsection{Demodulation of frequency-carrier composite color pattern}

With the snapshot acquired frequency-carrier composite color pattern, the demodulation is required to separate the phase-shifting patterns in $x$ and $y$ directions from the single-shot pattern. Based on the color separation, the figure data in $\mathrm{RB}$ channels can be separated to obtain the composite patterns $x$ and $y$ directions, respectively. Without loss of generality, we take the composite pattern in $x$ direction as the case for analysis.

In Subsection 2.2, the phase-shifting patterns are modulated by frequency carrier patterns with diverse frequencies. The band-pass filters can be applied to separate the diverse modulated patterns with different frequencies $f_{f x, n}$ in each direction, in which the multi-step phase-shifting patterns are integrated. The band-pass filters are designed centered at $f_{f x, n}$ to 
achieve the uniform filtering for the phase-shifting data. The band-pass filters based on the Butterworth filter are adopted in our method, which can achieve smoother transition and minimal side-lobe ripple effect. Based on the band-pass filters, four composite patterns can be obtained for the latter processing. Then, the low-pass filter in the direction of frequency carrier pattern is applied to obtain the phase-shifting figures. It should be noted that, the cut-off frequency should be set as close as possible to the lower cut-off frequency of the band-pass filters. After that, the phase-shifting patterns in $x$ direction can be demodulated. With the eight demodulated phase-shifting patterns in $x$ and $y$ directions, the transmitted wavefront from test object can be reconstructed with phase-shifting deflectometry. The flow diagram for the proposed instantaneous deflectometric wavefront measurement is summarized in Fig. 3.

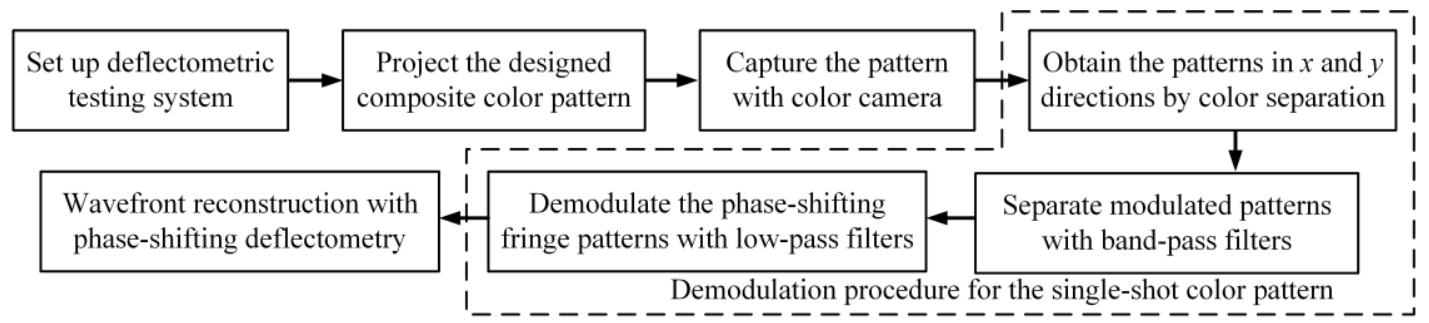

Fig. 3. Flow diagram for the proposed instantaneous deflectometric wavefront measurement.

\section{SIMULATION RESULTS}

To validate the feasibility of the proposed method, the numerical simulation was performed, in which an additional distortion was added in the phase-shifting patterns as the induced wavefront aberration. The carrier frequencies in $x$ and $y$ directions are both set to 40,70,100 and 130 cycles for the measurement field size $1080 \times 1080$ pixels, and there are about 12 cycles in each phase-shifting fringe pattern.

Figure 4 shows the demodulation result of the single-shot composite color pattern, with which the demodulated phaseshifting patterns in $x$ and $y$ directions can be obtained to reconstruct the phase maps. The retrieved wrapped phase maps in $x$ and $y$ directions are shown in Figs. 5(a) and 5(d), and the corresponding unwrapped phase maps are shown in Figs. 5(b) and 5(e), respectively. Figure 5(c) and 5(f) are the residual errors of the retrieved phase maps with respect to the true values in $x$ and $y$ directions, whose root-mean-square (RMS) values are $0.0025 \mathrm{rad}$ and $0.0080 \mathrm{rad}$, respectively.

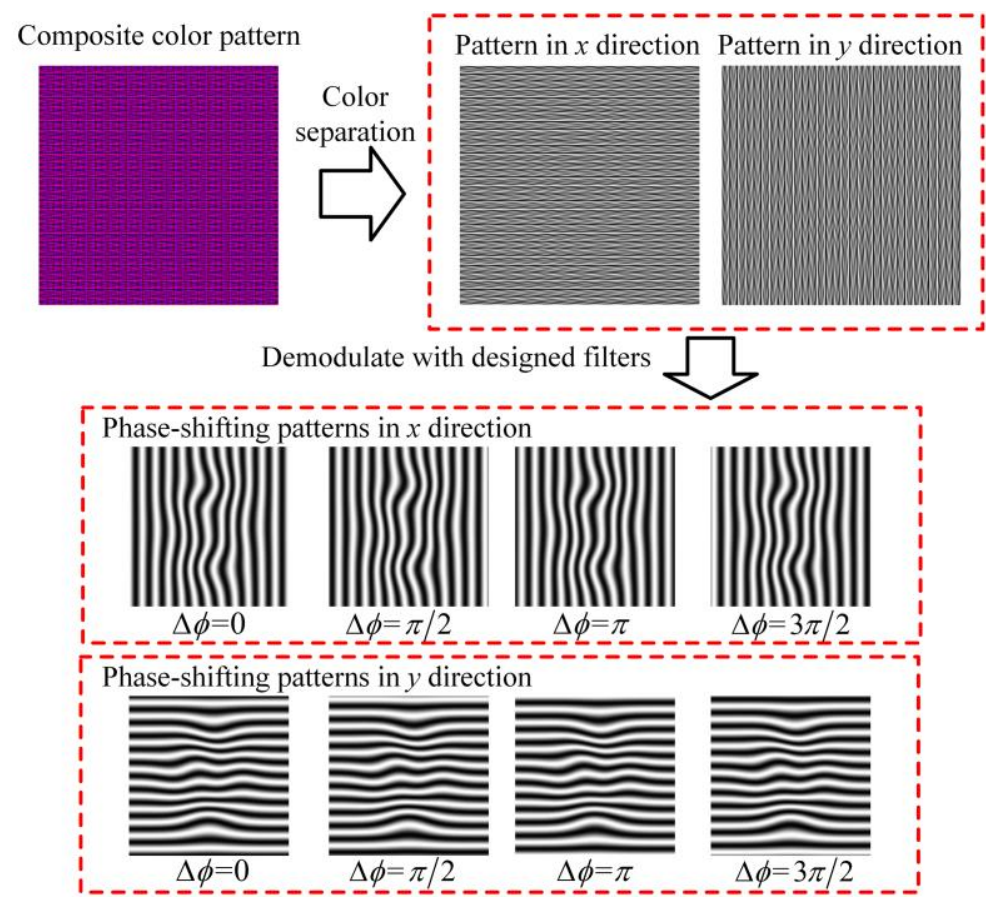

Fig. 4. Demodulation results of the distorted phase-shifting patterns in simulation. 

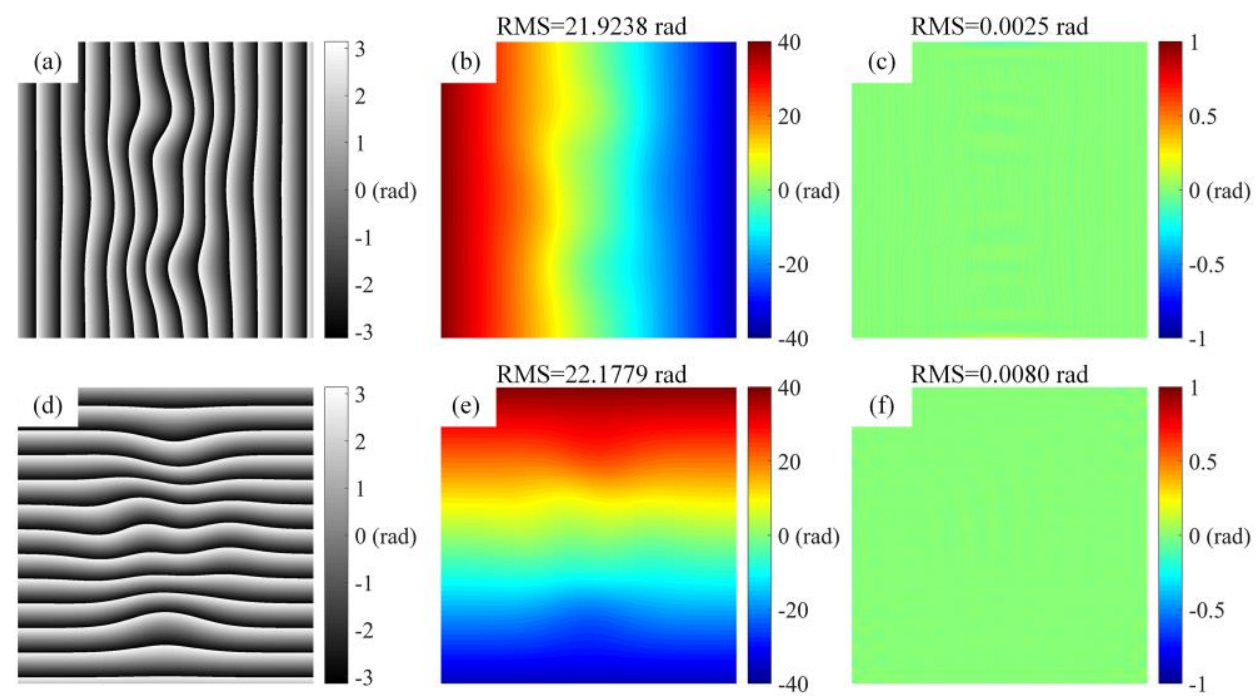

Fig. 5. Phase retrieve for the demodulated patterns in the simulation. Retrieved wrapped phase map in (a) $x$ and (d) $y$ directions; unwrapped phase map in (b) $x$ and (e) $y$ directions; Phase differences (c) and (f) of (b) and (e) relative to the true values in $x$ and $y$ directions, respectively.

Additionally, the Gaussian white noise with a signal-to-noise ratio (SNR) of $25 \mathrm{~dB}$ is added to analysis the robustness of the proposed method. The phase residual errors between the retrieved unwrapped phase maps and the true values in $x$ and $y$ directions are shown in Figs. 6(a) and 6(b), with the RMS value being 0.0713 rad and 0.0710 rad, respectively. According to Figs. 5 and 6, the proposed method provides a feasible and efficient way for the instantaneous wavefront measurement.
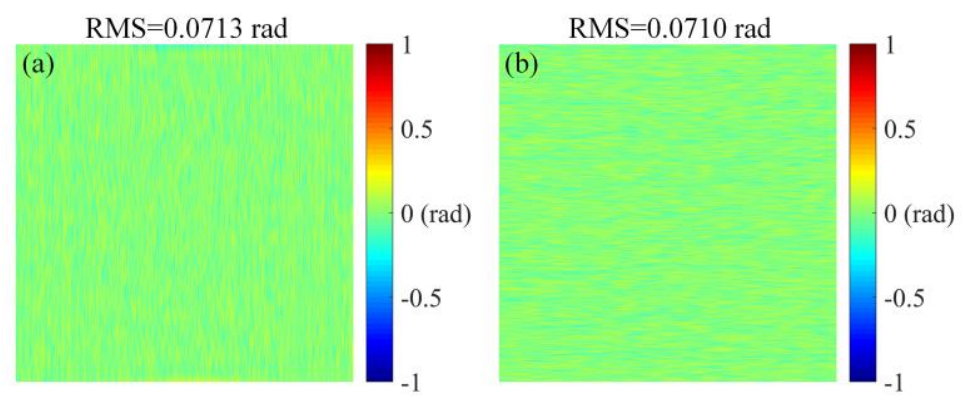

Fig. 6. Phase differences between the retrieved phase and true value with the existence of noise: (a) $x$ and (b) $y$ directions.

\section{EXPERIMENTAL RESULTS}

According to Fig. 1, an experimental instantaneous deflectometric system for wavefront measurement has been built to demonstrate the feasibility of the proposed method. The pixel number of LCD screen for fringe projection is $1920(\mathrm{H}) \times$ $1080(\mathrm{~V})$, with the corresponding pixel size being $0.265 \mathrm{~mm}(\mathrm{H}) \times 0.265 \mathrm{~mm}(\mathrm{~V})$. The image acquisition device is a three-channel 8-bit color CCD camera with $1348(\mathrm{H}) \times 1280(\mathrm{~V})$ pixels resolution and $3.63 \mu \mathrm{m}(\mathrm{H}) \times 3.63 \mu \mathrm{m}(\mathrm{V})$ pixel size. The test object is a convex lens with $25.4 \mathrm{~mm}$ in diameter and 1.51 in refractive index.

Figure 7(a) shows the captured composite color pattern after passing through the test object; Figs. 7(b) and 7(d) are the extracted composite patterns from RB channels with the phase-shifting information in $x$ and $y$ directions, respectively. Based on the demodulation method introduced in Subsection 2.3, the fringe patterns in $x$ and $y$ directions can be obtained, as are shown in Figs. 7(c) and 7(e). Then, the demodulated phase-shifting patterns can be used to retrieve unwrapped phases with the phase-shifting algorithm, based on which the test wavefront can be reconstructed with phase-shifting deflectometry. Figure 8 shows the measured wavefront with the proposed method, whose RMS value is $0.7559 \mu \mathrm{m}$. 


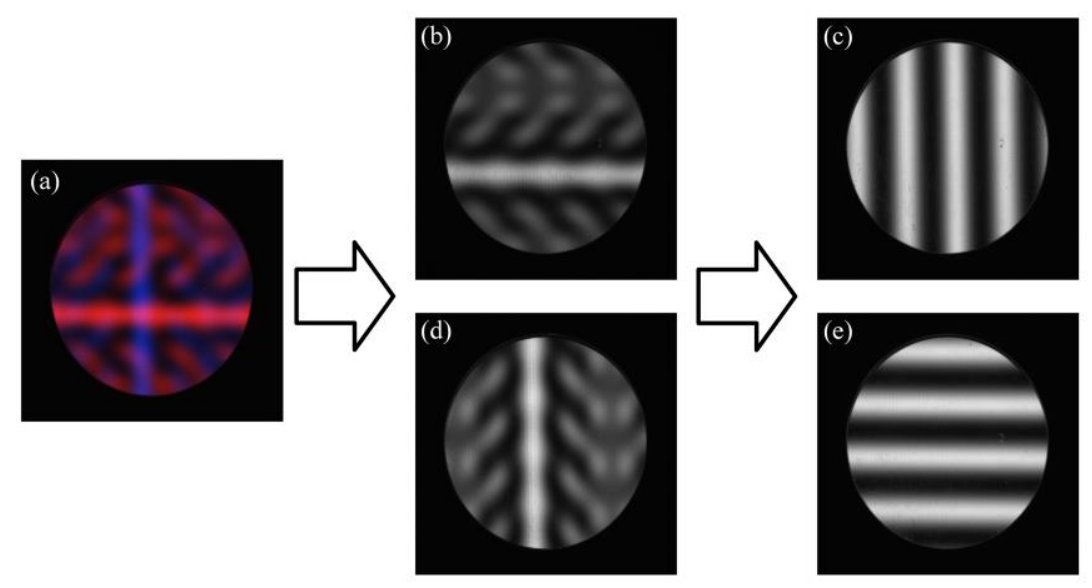

Fig. 7. Demodulation of captured composite color pattern in experiment. (a) Captured composite color pattern; (b) and (d) are the composite patterns in $x$ and $y$ directions after color separation, respectively, (c) and (e) are the corresponding demodulated phase-shifting patterns.

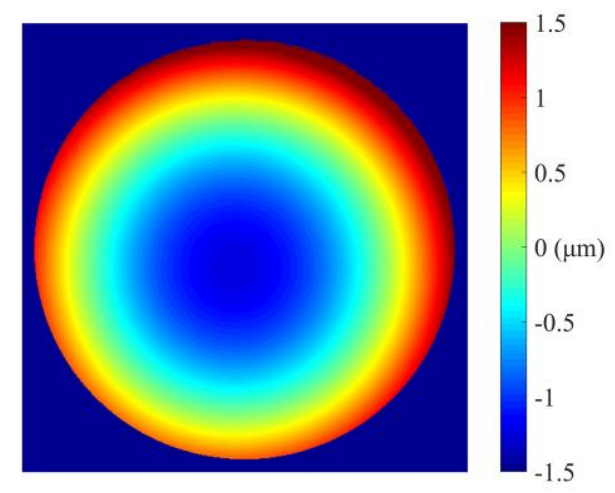

Fig. 8. Measured wavefront in the experiment.

\section{CONCLUSION}

In this paper, an instantaneous method based on deflectometry is proposed for wavefront measurement. The instantaneous measurement is realized by the frequency carrier composite and color-encoded method, in which the multiple phase-shifting fringe patterns for projection are modulated into a single composite color pattern. With the demodulated results of phase-shifting patterns in orthogonal directions, the traditional phase-shifting deflectometry can be applied to reconstruct the measured wavefront. Both the numerical simulation and experiment have been performed to validate the feasibility of the proposed instantaneous deflectometric wavefront measurement method. The proposed method can achieve the measurement of transmitted wavefront with a single shot. In addition, the multiple phase-shifting patterns in the same direction are coupled into one color channel, and it avoids the crosstalk among various color channels and photon response nonuniformity. Thus, the proposed testing method provides a feasible way to achieve the rapid, transient wavefront measurement with large dynamic range, minimizing the environmental disturbance.

\section{ACKNOWLEDGEMENTS}

The activities of this work are supported by National Natural Science Foundation of China (NSFC) (51775528), Guangxi Key Laboratory of Optoelectroric Information Processing (GD18205), Zhejiang Provincial Natural Science Foundation of China (LY17E050014), China Postdoctoral Science Foundation (2017M621928), Zhejiang Key Discipline of Instrument Science and Technology (JL150508). 


\section{REFERENCES}

[1] Steimle, L. J. and Wang, Y., "Development of a broadband achromatic Twyman-Green interferometer," Optical Engineering 33(3), 771-775 (1997).

[2] Asfour, J.-M. and Poleshchuk, A. G., "Asphere testing with a Fizeau interferometer based on a combined computer-generated hologram," Journal of the Optical Society of America. A 23(1), 173-178 (2006).

[3] Wang, D., Yang, Y., Chen, C. and Zhuo, Y., "Point diffraction interferometer with adjustable fringe contrast for testing spherical surfaces," Applied Optics 50(16), 2342-2348 (2011).

[4] Yang, Z., Dou, J., Yuan, Q. and Gao, Z., "Lensless phase-shifting point diffraction interferometer for spherical mirror measurement," Optics and Lasers in Engineering 107, 119-126 (2018).

[5] Faber, C., Olesch, E., Krobot, R. and Hausler, G., "Deflectometry challenges interferometry- the competition gets tougher," Proc. SPIE 8493, 84930R (2012).

[6] Su, P., Wang, S., Khreishi, M., Wang, Y. and Burge, J. H., "SCOTS: a reverse hartmann test with high dynamic range for giant Magellan telescope primary mirror segments," Proc. SPIE 8450, 84500W (2012).

[7] Muñoz-Potosi, A. F., Granados-Agustín, F., Campos-García, M., Valdivieso-González, L. G. and PercinoZacarias, M. E., "Deflectometry using a Hartmann screen to measure tilt, decentering and focus errors in a spherical surface," Optics Communications 402, 375-381(2017).

[8] Wang, D., Zhang, S., Wu, R., Huang, C. Y., Cheng, H.-N. and Liang, R., "Computer-aided high-accuracy testing of reflective surface with reverse Hartmann test," Optics Express 24(17), 19671-19681 (2016).

[9] Huang, R., Su, P., Horne, T., Zappellini, G. B. and Burge, J. H., "Measurement of a large deformable aspherical mirror using SCOTS (Software Configurable Optical Test System)," Proc. SPIE 8838, 883807 (2013).

[10] Knauer, M. C., Kaminski, J. and Hausler, G., "Phase measuring deflectometry: a new approach to measure specular freeform surface," Proc. SPIE 5457, 366-376 (2004).

[11] Huang, R., Su, P., Burge, J. H., Huang, L. and Idir, M., "High-accuracy aspheric x-ray mirror metrology using software configurable optical testing system/deflectometry," Optical Engineering 54(8), 084103 (2015).

[12] Geng, J., "Structured-light 3D surface imaging: a tutorial," Advances in Optics and Photonics 3, 128-160 (2011).

[13] Takeda, M. and Mutoh, K., "Fourier transform profilometry for the automatic measurement 3-D object shapes," Applied Optics 22(24), 3977-3982 (1983).

[14]Zhang, Z., Towers, C. E. and Towers, D. P., "Time efficient color fringe projection system for 3D shape and color using optimum 3-frequency Selection," Optics Express 14(14), 6444-6455 (2006).

[15] Trumper, I., Choi, H. and Kim, D. W., "Instantaneous phase mapping deflectometry for dynamic deformable mirror characterization," Proc. SPIE 10401, 104010S (2017).

[16] Wang, D., Gong, Z., Xu, P., Liang, R., Kong, M. and Zhao, J., "Accurate calibration of geometrical error in reflective surface testing based on reverse Hartmann test," Optics Express 26(7), 8113-8124 (2018).

[17] Wang, D., Xu, P., Gong, Z., Xie, Z., Liang, R., Xu, X., Kong, M. and Zhao, J., "Transmitted wavefront testing with large dynamic range based on computer-aided deflectometry," Journal of Optics 20(6), 065705 (2018).

[18] Schreiber, H., and Bruning, J. H., [Optical Shop Testing], John Wiley \& Sons Inc, 568-596, New Jersey (2007). 